\title{
Schur-convexity for compositions of complete symmetric function dual
}

\author{
Huan-Nan Shi ${ }^{1}$, Pei Wang ${ }^{1}$ and Jian Zhang ${ }^{1 *}$
}

"Correspondence:

13601356317@139.com

'Basic Teaching Department,

Teachers' College, Beijing Union

University, Beijing, P.R. China

\begin{abstract}
The Schur-convexity for certain compound functions involving the dual of the complete symmetric function is studied. As an application, the Schur-convexity of some special symmetric functions is discussed and some inequalities are established.

MSC: 05E05; 26B25

Keywords: Schur-convexity; Schur-geometric convexity; Schur-harmonic convexity; Completely symmetric function; Dual form
\end{abstract}

\section{Introduction}

Throughout the article, $\mathbb{R}$ denotes the set of real numbers, $\boldsymbol{x}=\left(x_{1}, x_{2}, \ldots, x_{n}\right)$ denotes $n$ tuple ( $n$-dimensional real vectors), the set of vectors can be written as

$$
\begin{aligned}
& \mathbb{R}^{n}=\left\{\boldsymbol{x}=\left(x_{1}, x_{2}, \ldots, x_{n}\right): x_{i} \in \mathbb{R}, i=1,2, \ldots, n\right\}, \\
& \mathbb{R}_{+}^{n}=\left\{\boldsymbol{x}=\left(x_{1}, x_{2}, \ldots, x_{n}\right): x_{i}>0, i=1,2, \ldots, n\right\}, \\
& \mathbb{R}_{-}^{n}=\left\{\boldsymbol{x}=\left(x_{1}, x_{2}, \ldots, x_{n}\right): x_{i}<0, i=1,2, \ldots, n\right\} .
\end{aligned}
$$

In particular, the notations $\mathbb{R}$ and $\mathbb{R}_{+}$denote $\mathbb{R}^{1}$ and $\mathbb{R}_{+}^{1}$, respectively.

In recent years, the Schur-convexity, Schur-geometric, and Schur-harmonic convexities of various symmetric functions have been a hot topic of inequality research [1-30].

The following complete symmetric function is an important class of symmetric functions.

For $\boldsymbol{x}=\left(x_{1}, x_{2}, \ldots, x_{n}\right) \in \mathbb{R}^{n}$, the complete symmetric function $c_{n}(\boldsymbol{x}, r)$ is defined as

$$
c_{n}(\boldsymbol{x}, r)=\sum_{i_{1}+i_{2}+\cdots+i_{n}=r} x_{1}^{i_{1}} x_{2}^{i_{2}} \cdots x_{n}^{i_{n}}
$$

where $c_{0}(\boldsymbol{x}, r)=1, r \in\{1,2, \ldots, n\}, i_{1}, i_{2}, \ldots, i_{n}$ are nonnegative integers.

It has been investigated by many mathematicians, and there are many interesting results in the literature.

Guan [4] discussed the Schur-convexity of $c_{n}(\boldsymbol{x}, r)$ and proved the following.

(c) The Author(s) 2020. This article is licensed under a Creative Commons Attribution 4.0 International License, which permits use, sharing, adaptation, distribution and reproduction in any medium or format, as long as you give appropriate credit to the original author(s) and the source, provide a link to the Creative Commons licence, and indicate if changes were made. The images or other third party material in this article are included in the article's Creative Commons licence, unless indicated otherwise in a credit line to the material. If material is not included in the article's Creative Commons licence and your intended use is not permitted by statutory regulation or exceeds the permitted use, you will need to obtain permission directly from the copyright holder. To view a copy of this licence, visit http://creativecommons.org/licenses/by/4.0/. 
Proposition $1 c_{n}(\boldsymbol{x}, r)$ is increasing and Schur-convex on $\mathbb{R}_{+}^{n}$.

Subsequently, Chu et al. [1] proved the following.

Proposition $2 c_{n}(\boldsymbol{x}, r)$ is Schur-geometrically convex and Schur-harmonically convex on $\mathbb{R}_{+}^{n}$.

In 2016, Shi et al. [18] further considered the Schur-convexity of $c_{n}(\boldsymbol{x}, r)$ on $\mathbb{R}_{-}^{n}$, which proved the following proposition.

Proposition 3 If $r$ is an even integer (or odd integer, respectively), then $c_{n}(\boldsymbol{x}, r)$ is decreasing and Schur-convex (or increasing and Schur-concave, respectively) on $\mathbb{R}_{-}^{n}$.

The dual form of the complete symmetric function $c_{n}(\boldsymbol{x}, r)$ is defined as

$$
c_{n}^{*}(\boldsymbol{x}, r)=\prod_{i_{1}+i_{2}+\cdots+i_{n}=r} \sum_{j=1}^{n} i_{j} x_{j}
$$

where $c_{0}^{*}(\boldsymbol{x}, r)=1, r \in\{1,2, \ldots, n\}, i_{1}, i_{2}, \ldots, i_{n}$ are nonnegative integers.

Zhang and Shi [17] proved the following two propositions.

Proposition 4 For $r=1,2, \ldots, n, c_{n}^{*}(\boldsymbol{x}, r)$ is increasing and Schur-concave on $\mathbb{R}_{+}^{n}$.

Proposition 5 For $r=1,2, \ldots, n, c_{n}^{*}(\boldsymbol{x}, r)$ is Schur-geometrically convex and Schurharmonically convex on $\mathbb{R}_{+}^{n}$.

Notice that

$$
c_{n}^{*}(-\mathbf{x}, r)=(-1)^{r} c_{n}^{*}(\mathbf{x}, r),
$$

it is not difficult to prove the following proposition.

Proposition 6 If $r$ is an even integer (or odd integer, respectively), then $c_{n}^{*}(\boldsymbol{x}, r)$ is decreasing and Schur-concave (or increasing and Schur-convex, respectively) on $\mathbb{R}_{-}^{n}$.

In this paper we will study the Schur-convexity, Schur-geometric and Schur-harmonic convexities of the following composite function of $c_{n}^{*}(\boldsymbol{x}, r)$ :

$$
c_{n}^{*}(f(\mathbf{x}), r)=c_{n}^{*}\left(f\left(x_{1}\right), f\left(x_{2}\right), \ldots, f\left(x_{n}\right), r\right)=\prod_{i_{1}+i_{2}+\cdots+i_{n}=r} \sum_{j=1}^{n} i_{j}\left(f\left(x_{j}\right)\right),
$$

where $f$ is a positive function which satisfies certain conditions.

Our main results are as follows.

Theorem 1 Let $I \subset \mathbb{R}$ be a symmetric convex set with nonempty interior, and let $f: I \rightarrow \mathbb{R}_{+}$ be continuous on I and differentiable in the interior of $I$.

(a) Iff is a log-convex function on $I$, then for any $r=1,2, \ldots, n, c_{n}^{*}(f(\mathbf{x}), r)$ is a Schur-convex function on $I^{n}$; 
(b) Iff is a concave function on $I$, then for any $r=1,2, \ldots, n, c_{n}^{*}(f(\mathbf{x}), r)$ is a Schur-concave function on $I^{n}$.

Theorem 2 Let $I \subset \mathbb{R}_{+}$be a symmetric convex set with nonempty interior and let $f: I \rightarrow \mathbb{R}_{+}$ be continuous on I and differentiable in the interior of I.

(a) Iff is an increasing and log-convex function on $I$, then for any $r=1,2, \ldots, n$, $c_{n}^{*}(f(\boldsymbol{x}), r)$ is a Schur-geometrically convex function on $I^{n}$.

(b) Iff is a descending and concave function on $I$, then for any $r=1,2, \ldots, n, c_{n}^{*}(f(\boldsymbol{x}), r)$ is a Schur-geometrically concave function on $I^{n}$.

Theorem 3 Let $I \subset \mathbb{R}_{+}$be a symmetric convex set with nonempty interior, and let $f: I \rightarrow$ $\mathbb{R}_{+}$be continuous on $I$ and differentiable in the interior of $I$.

(a) If $f$ is an increasing and log-convex function on $I$, then for any $r=1,2, \ldots, n$, $c_{n}^{*}(f(\boldsymbol{x}), r)$ is a Schur-harmonically convex function on $I^{n}$.

(b) Iff is a descending and concave function on $I$, then for any $r=1,2, \ldots, n, c_{n}^{*}(f(\boldsymbol{x}), r)$ is a Schur-harmonically concave function on $I^{n}$.

\section{Definitions and lemmas}

For convenience, we introduce some definitions as follows.

Definition $1([31,32])$ Let $\boldsymbol{x}=\left(x_{1}, x_{2}, \ldots, x_{n}\right)$ and $\boldsymbol{y}=\left(y_{1}, y_{2}, \ldots, y_{n}\right) \in \mathbb{R}^{n}$.

(a) $\boldsymbol{x} \geq \boldsymbol{y}$ means $x_{i} \geq y_{i}$ for all $i=1,2, \ldots, n$.

(b) Let $\Omega \subset \mathbb{R}^{n}, \varphi: \Omega \rightarrow \mathbb{R}$ is said to be increasing if $\boldsymbol{x} \geq \boldsymbol{y}$ implies $\varphi(\boldsymbol{x}) \geq \varphi(\boldsymbol{y})$. $\varphi$ is said to be decreasing if and only if $-\varphi$ is increasing.

Definition $2([31,32])$ Let $\boldsymbol{x}=\left(x_{1}, x_{2}, \ldots, x_{n}\right)$ and $\boldsymbol{y}=\left(y_{1}, y_{2}, \ldots, y_{n}\right) \in \mathbb{R}^{n}$.

(a) $\boldsymbol{x}$ is said to be majorized by $\boldsymbol{y}$ (in symbols $\boldsymbol{x} \prec \boldsymbol{y}$ ) if $\sum_{i=1}^{k} x_{[i]} \leq \sum_{i=1}^{k} y_{[i]}$ for $k=1,2, \ldots, n-1$ and $\sum_{i=1}^{n} x_{i}=\sum_{i=1}^{n} y_{i}$, where $x_{[1]} \geq x_{[2]} \geq \cdots \geq x_{[n]}$ and $y_{[1]} \geq y_{[2]} \geq \cdots \geq y_{[n]}$ are rearrangements of $\boldsymbol{x}$ and $\boldsymbol{y}$ in a descending order.

(b) Let $\Omega \subset \mathbb{R}^{n}, \varphi: \Omega \rightarrow \mathbb{R}$ is said to be a Schur-convex function on $\Omega$ if $\boldsymbol{x} \prec \boldsymbol{y}$ on $\Omega$ implies $\varphi(\boldsymbol{x}) \leq \varphi(\boldsymbol{y}) . \varphi$ is said to be a Schur-concave function on $\Omega$ if and only if $-\varphi$ is Schur-convex function on $\Omega$.

Definition $3([31,32])$ Let $\boldsymbol{x}=\left(x_{1}, x_{2}, \ldots, x_{n}\right)$ and $\boldsymbol{y}=\left(y_{1}, y_{2}, \ldots, y_{n}\right) \in \mathbb{R}^{n}$.

(a) $\Omega \subset \mathbb{R}^{n}$ is said to be a convex set if $\boldsymbol{x}, \boldsymbol{y} \in \Omega, 0 \leq \alpha \leq 1$, implies $\alpha \mathbf{x}+(1-\alpha) \boldsymbol{y}=\left(\alpha x_{1}+(1-\alpha) y_{1}, \alpha x_{2}+(1-\alpha) y_{2}, \ldots, \alpha x_{n}+(1-\alpha) y_{n}\right) \in \Omega$.

(b) Let $\Omega \subset \mathbb{R}^{n}$ be a convex set. A function $\varphi: \Omega \rightarrow \mathbb{R}$ is said to be a convex function on $\Omega$ if

$$
\varphi(\alpha \mathbf{x}+(1-\alpha) \boldsymbol{y}) \leq \alpha \varphi(\mathbf{x})+(1-\alpha) \varphi(\boldsymbol{y})
$$

for all $\boldsymbol{x}, \boldsymbol{y} \in \Omega$, and all $\alpha \in[0,1]$. $\varphi$ is said to be a concave function on $\Omega$ if and only if $-\varphi$ is a convex function on $\Omega$.

Definition $4([31,32])$

(a) A set $\Omega \subset \mathbb{R}^{n}$ is called a symmetric set if $\boldsymbol{x} \in \Omega$ implies $\mathbf{x} P \in \Omega$ for every $n \times n$ permutation matrix $P$. 
(b) A function $\varphi: \Omega \rightarrow \mathbb{R}$ is called symmetric if, for every permutation matrix $P$, $\varphi(\mathbf{x} P)=\varphi(\mathbf{x})$ for all $\boldsymbol{x} \in \Omega$.

Lemma 1 (Schur-convex function decision theorem [31,32]) Let $\Omega \subset \mathbb{R}^{n}$ be symmetric and have a nonempty interior convex set. $\Omega^{0}$ is the interior of $\Omega . \varphi: \Omega \rightarrow \mathbb{R}$ is continuous on $\Omega$ and differentiable in $\Omega^{0}$. Then $\varphi$ is the Schur-convex (or Schur-concave, respectively) function if and only if $\varphi$ is symmetric on $\Omega$ and

$$
\left(x_{1}-x_{2}\right)\left(\frac{\partial \varphi}{\partial x_{1}}-\frac{\partial \varphi}{\partial x_{2}}\right) \geq 0(\text { or } \leq 0, \text { respectively })
$$

holds for any $\mathbf{x} \in \Omega^{0}$.

The first systematical study of the functions preserving the ordering of majorization was made by Issai Schur in 1923. In Schur's honor, such functions are said to be "Schurconvex". They can be used extensively in analytic inequalities, combinatorial optimization, quantum physics, information theory, and other related fields. See [31].

Definition 5 ([33]) Let $\boldsymbol{x}=\left(x_{1}, x_{2}, \ldots, x_{n}\right) \in \mathbb{R}_{+}^{n}$ and $\boldsymbol{y}=\left(y_{1}, y_{2}, \ldots, y_{n}\right) \in \mathbb{R}_{+}^{n}$.

(a) $\Omega \subset \mathbb{R}_{+}^{n}$ is called a geometrically convex set if $\left(x_{1}^{\alpha} y_{1}^{\beta}, x_{2}^{\alpha} y_{2}^{\beta}, \ldots, x_{n}^{\alpha} y_{n}^{\beta}\right) \in \Omega$ for all $\boldsymbol{x}$, $\boldsymbol{y} \in \Omega$ and $\alpha, \beta \in[0,1]$ such that $\alpha+\beta=1$.

(b) Let $\Omega \subset \mathbb{R}_{+}^{n}$. The function $\varphi: \Omega \rightarrow \mathbb{R}_{+}$is said to be a Schur-geometrically convex function on $\Omega$ if $\left(\log x_{1}, \log x_{2}, \ldots, \log x_{n}\right) \prec\left(\log y_{1}, \log y_{2}, \ldots, \log y_{n}\right)$ on $\Omega$ implies $\varphi(\boldsymbol{x}) \leq \varphi(\boldsymbol{y})$. The function $\varphi$ is said to be a Schur-geometrically concave function on $\Omega$ if and only if $-\varphi$ is a Schur-geometrically convex function on $\Omega$.

The Schur-geometric convexity was proposed by Zhang [33] in 2004, and it was investigated by Chu et al. [34], Guan [35], Sun et al. [36], and so on. We also note that some authors use the term "Schur multiplicative convexity".

In 2009, Chu ([1, 2, 37]) introduced the notion of Schur-harmonically convex function, and some interesting inequalities were obtained.

Definition 6 ([37]) Let $\Omega \subset \mathbb{R}_{+}^{n}$ or $\Omega \subset \mathbb{R}_{-}^{n}$.

(a) A set $\Omega$ is said to be harmonically convex if $\frac{\boldsymbol{x y}}{\lambda \boldsymbol{x}+(1-\lambda) \boldsymbol{y}} \in \Omega$ for every $\boldsymbol{x}, \boldsymbol{y} \in \Omega$ and $\lambda \in[0,1]$, where $\boldsymbol{x y}=\sum_{i=1}^{n} x_{i} y_{i}$ and $\frac{1}{\boldsymbol{x}}=\left(\frac{1}{x_{1}}, \frac{1}{x_{2}}, \ldots, \frac{1}{x_{n}}\right)$.

(b) A function $\varphi: \Omega \rightarrow \mathbb{R}_{+}$is said to be Schur-harmonically convex on $\Omega$ if $\frac{1}{x} \prec \frac{1}{y}$ implies $\varphi(\boldsymbol{x}) \leq \varphi(\boldsymbol{y})$. A function $\varphi$ is said to be a Schur-harmonically concave function on $\Omega$ if and only if $-\varphi$ is a Schur-harmonically convex function.

Remark 1 We extend the definition and determination theorem of Schur-harmonically convex function established by Chu as follows:

(a) $\Omega \subset \mathbb{R}_{+}^{n}$ is extended to $\Omega \subset \mathbb{R}_{+}^{n}$ or $\Omega \subset \mathbb{R}_{-}^{n}$;

(b) The function $\varphi: \Omega \rightarrow \mathbb{R}$ must not be a positive function.

Lemma $2([31,32])$ Let the set $\mathbb{A}, \mathbb{B} \subset \mathbb{R}, \varphi: \mathbb{B}^{n} \rightarrow \mathbb{R}, f: \mathbb{A} \rightarrow \mathbb{B}$ and $\psi\left(x_{1}, x_{2}, \ldots, x_{n}\right)=$ $\varphi\left(f\left(x_{1}\right), f\left(x_{2}\right), \ldots, f\left(x_{n}\right)\right): \mathbb{A}^{n} \rightarrow \mathbb{R}$.

(a) Iff is convex and $\varphi$ is increasing and Schur-convex, then $\psi$ is Schur-convex;

(b) Iff is concave, $\varphi$ is increasing and Schur-concave, then $\psi$ is Schur-concave. 
Lemma 3 Let the set $\Omega \subset \mathbb{R}_{+}^{n}$. The function $\varphi: \Omega \rightarrow \mathbb{R}_{+}$is differentiable.

(a) If $\varphi$ is increasing and Schur-convex, then $\varphi$ is Schur geometrically convex.

(b) If $\varphi$ is decreasing and Schur-concave, then $\varphi$ is Schur geometrically concave.

Lemma 4 Let the set $\Omega \subset \mathbb{R}_{+}^{n}$. The function $\varphi: \Omega \rightarrow \mathbb{R}_{+}$is differentiable.

(a) If $\varphi$ is increasing and Schur-convex, then $\varphi$ is Schur-harmonically convex.

(b) If $\varphi$ is decreasing and Schur-concave, then $\varphi$ is Schur-harmonically concave.

Lemma $5([31,32])$ Let $\left(\boldsymbol{x}=\left(x_{1}, x_{2}, \ldots, x_{n}\right) \in \mathbb{R}^{n}\right.$. Then

$$
(A(\boldsymbol{x}), A(\mathbf{x}), \ldots, A(\boldsymbol{x})) \prec\left(\boldsymbol{x}=\left(x_{1}, x_{2}, \ldots, x_{n}\right),\right.
$$

where $A(\mathbf{x})=\frac{1}{n} \sum_{i}^{n} x_{i}$.

Lemma 6 ([22]) Let

$$
q(t)=\frac{u^{t}-1}{t}
$$

If $u>1$, then $q(t)$ is a log-convex function on $\mathbb{R}_{+}$.

\section{Proof of main results}

Proof of Theorem 1 For the case of $r=1$ and $r=2$, it is easy to prove that $c_{n}^{*}(f(\boldsymbol{x}), r)$ is Schur-convex on $I^{n}$.

Now consider the case of $r \geq 3$. By the symmetry of $c_{n}^{*}(f(\boldsymbol{x}), r)$, without loss of generality, we can set $x_{1}>x_{2}$.

$$
\begin{aligned}
c_{n}^{*}((\boldsymbol{x}), r)= & \prod_{\substack{i_{1}+i_{2}+\cdots+i_{n}=r \\
i_{1} \neq 0, i_{2}=0}} \sum_{j=1}^{n} i_{j} f\left(x_{j}\right) \times \prod_{\substack{i_{1}+i_{2}+\cdots+i_{n}=r \\
i_{1}=0, i_{2} \neq 0}} \sum_{j=1}^{n} i_{j} f\left(x_{j}\right) \\
& \times \prod_{\substack{i_{1}+i_{2}+\cdots+i_{n}=r \\
i_{1} \neq 0, i_{2} \neq 0}} \sum_{j=1}^{n} i_{j} f\left(x_{j}\right) \times \prod_{\substack{i_{1}+i_{2}+\cdots+i_{n}=r \\
i_{1}=0, i_{2}=0}} \sum_{j=1}^{n} i_{j} f\left(x_{j}\right) .
\end{aligned}
$$

Then

$$
\begin{aligned}
\frac{\partial c_{n}^{*}(f(\boldsymbol{x}), r)}{\partial x_{1}}= & c_{n}^{*}(f(\mathbf{x}), r) \\
& \times\left(\sum_{\substack{i_{1}+i_{2}+\cdots+i_{n}=r \\
i_{1} \neq 0, i_{2}=0}} \frac{i_{1} f^{\prime}\left(x_{1}\right)}{\sum_{j=1}^{n} i_{j} f\left(x_{j}\right)}+\sum_{\substack{i_{1}+i_{2}+\cdots+i_{n}=r \\
i_{1} \neq 0, i_{2} \neq 0}} \frac{i_{1} f^{\prime}\left(x_{1}\right)}{\sum_{j=1}^{n} i_{j} f\left(x_{j}\right)}\right) \\
= & c_{n}^{*}(f(\boldsymbol{x}), r)\left(\sum_{\substack{k+k_{3}+\cdots+k_{n}=r \\
k \neq 0}} \frac{k f^{\prime}\left(x_{1}\right)}{k f\left(x_{1}\right)+\sum_{j=3}^{n} i_{j} f\left(x_{j}\right)}\right. \\
& \left.+\sum_{\substack{k+m+i_{3}+\cdots+i_{n}=r \\
k \neq 0, m \neq 0}} \frac{k f^{\prime}\left(x_{1}\right)}{k f\left(x_{1}\right)+m f\left(x_{2}\right)+\sum_{j=3}^{n} i_{j} f\left(x_{j}\right)}\right) .
\end{aligned}
$$


By the same arguments,

$$
\begin{aligned}
\frac{\partial c_{n}^{*}(f(\mathbf{x}), r)}{\partial x_{2}}= & c_{n}^{*}(f(\mathbf{x}), r) \\
= & c_{n}^{*}(f(\mathbf{x}), r)\left(\sum_{\substack{k+k_{3}+\cdots+k_{n}=r \\
k \neq 0}} \frac{k f^{\prime}\left(x_{2}\right)}{k f\left(x_{2}\right)+\sum_{j=3}^{n} i_{j} f\left(x_{j}\right)}\right. \\
& \left.+\sum_{\substack{k+m+i_{3}+\cdots+i_{n}=r \\
k \neq 0, m \neq 0}} \frac{k f^{\prime}\left(x_{2}\right)}{k f\left(x_{2}\right)+m f\left(x_{1}\right)+\sum_{j=3}^{n} i_{j} f\left(x_{j}\right)}\right), \\
\frac{\partial c_{n}^{*}(f(\mathbf{x}), r)}{\partial x_{1}}- & \frac{\partial c_{n}^{*}(f(\mathbf{x}), r)}{\partial x_{2}}=c_{n}^{*}(f(\mathbf{x}), r)\left(A_{1}+A_{2}\right),
\end{aligned}
$$

where

$$
\begin{aligned}
A_{1} & =\sum_{\substack{k+k_{3}+\cdots+k_{n}=r \\
k \neq 0}}\left(\frac{k f^{\prime}\left(x_{1}\right)}{k f\left(x_{1}\right)+\sum_{j=3}^{n} i_{j} f\left(x_{j}\right)}-\frac{k f^{\prime}\left(x_{2}\right)}{k f\left(x_{2}\right)+\sum_{j=3}^{n} i_{j} f\left(x_{j}\right)}\right) \\
& =k \sum_{\substack{k+k_{3}+\cdots+k_{n}=r \\
k \neq 0}} \frac{k\left(f\left(x_{2}\right) f^{\prime}\left(x_{1}\right)-f\left(x_{1}\right) f^{\prime}\left(x_{2}\right)\right)+\left(f^{\prime}\left(x_{1}\right)-f^{\prime}\left(x_{2}\right)\right) \sum_{j=3}^{n} i_{j} f\left(x_{j}\right)}{\left(k f\left(x_{1}\right)+\sum_{j=3}^{n} i_{j} f\left(x_{j}\right)\right)\left(k f\left(x_{2}\right)+\sum_{j=3}^{n} i_{j} f\left(x_{j}\right)\right)}
\end{aligned}
$$

and

$$
\begin{aligned}
A_{2} & =\sum_{\substack{k+m+i_{3}+\cdots+i_{n}=r \\
k \neq 0, m \neq 0}}\left(\frac{k f^{\prime}\left(x_{1}\right)}{k f\left(x_{1}\right)+m f\left(x_{2}\right)+\sum_{j=3}^{n} i_{j} f\left(x_{j}\right)}-\frac{k f^{\prime}\left(x_{2}\right)}{k f\left(x_{2}\right)+m f\left(x_{1}\right)+\sum_{j=3}^{n} i_{j} f\left(x_{j}\right)}\right) \\
& =k \sum_{\substack{k+m+i_{3}+\cdots+i_{n}=r \\
k \neq 0, m \neq 0}} \frac{\delta}{\left(k f\left(x_{1}\right)+m f\left(x_{2}\right)+\sum_{j=3}^{n} i_{j} f\left(x_{j}\right)\right)\left(k f\left(x_{2}\right)+m f\left(x_{1}\right)+\sum_{j=3}^{n} i_{j} f\left(x_{j}\right)\right)}
\end{aligned}
$$

where

$$
\begin{aligned}
\delta= & k\left(f\left(x_{2}\right) f^{\prime}\left(x_{1}\right)-f\left(x_{1}\right) f^{\prime}\left(x_{2}\right)\right)+m\left(f\left(x_{1}\right) f^{\prime}\left(x_{1}\right)-f\left(x_{2}\right) f^{\prime}\left(x_{2}\right)\right) \\
& +\left(f^{\prime}\left(x_{1}\right)-f^{\prime}\left(x_{2}\right)\right) \sum_{j=3}^{n} i_{j} f\left(x_{j}\right) .
\end{aligned}
$$

(a) Since the log-convex function must be convex function, so $f^{\prime}\left(x_{1}\right)-f^{\prime}\left(x_{2}\right) \geq 0$ and $f\left(x_{2}\right) f^{\prime}\left(x_{1}\right)-f\left(x_{1}\right) f^{\prime}\left(x_{2}\right) \geq 0$, and since $\left(f(x) f^{\prime}(x)\right)^{\prime}=\left(f^{\prime}(x)\right)^{2}+f(x) f^{\prime \prime}(x) \geq 0$, so $f\left(x_{1}\right) f^{\prime}\left(x_{1}\right)-f\left(x_{2}\right) f^{\prime}\left(x_{2}\right) \geq 0$, and then $A_{1} \geq 0$ and $A_{2} \geq 0$. For $\boldsymbol{x} \in I^{n}$, we have

$$
\frac{\partial c_{n}^{*}(f(\boldsymbol{x}), r)}{\partial x_{1}}-\frac{\partial c_{n}^{*}(f(\mathbf{x}), r)}{\partial x_{2}} \geq 0
$$

by Lemma 1, it follows that $c_{n}^{*}(f(\boldsymbol{x}), r)$ is Schur-convex on $I^{n}$.

(b) By Proposition 4, we know that $c_{n}^{*}(\boldsymbol{x}, r)$ is increasing and Schur-concave on $\mathbb{R}_{+}^{n}$.

Since $f$ is concave, from (b) in Lemma 4 it follows that $c_{n}^{*}(f(\boldsymbol{x}), r)$ is Schur-concave on $I^{n}$.

The proof of Theorem 1 is completed. 
Proof of Theorem 2 Theorem 2 can be proved by Theorem 1 combined with Lemma 3.

The proof of Theorem 2 is completed.

Proof of Theorem 3 Theorem 3 can be proved by Theorem 1 combined with Lemma 4.

The proof of Theorem 3 is completed.

\section{Applications}

Let

$$
c_{n}^{*}\left(\frac{1}{\boldsymbol{x}}, r\right)=\prod_{i_{1}+i_{2}+\cdots+i_{n}=r} \sum_{j=1}^{n} i_{j}\left(\frac{1}{x_{j}}\right) .
$$

Theorem 4 The symmetric function $c_{n}^{*}\left(\frac{1}{x}, r\right)$ is Schur-convex on $\mathbb{R}_{+}^{n}$. If $r$ is an even integer (or odd integer, respectively), then $c_{n}^{*}\left(\frac{1}{\boldsymbol{x}}, r\right)$ is Schur-convex (or Schur-concave, respectively) on $\mathbb{R}_{-}^{n}$.

Proof Let $f(x)=\frac{1}{x}$. Then $(\ln f(x))^{\prime \prime}=\frac{1}{x^{2}}$, so $f(x)$ is $\log$-convex on $\mathbb{R}_{+}$, by (a) in Theorem 1 , it follows that $c_{n}^{*}\left(\frac{1}{\boldsymbol{x}}, r\right)$ is Schur-convex on $\mathbb{R}_{+}^{n}$.

For $\boldsymbol{x} \in \mathbb{R}_{-}^{n},-\boldsymbol{x} \in \mathbb{R}_{+}^{n}$, so $c_{n}^{*}\left(\frac{1}{-\boldsymbol{x}}, r\right)$ is Schur-convex on $\mathbb{R}_{-}^{n}$. But

$$
c_{n}^{*}\left(\frac{1}{-\boldsymbol{x}}, r\right)=(-1)^{r} c_{n}^{*}\left(\frac{1}{\boldsymbol{x}}, r\right) .
$$

This means that if $r$ is an even integer, then

$$
c_{n}^{*}\left(\frac{1}{\boldsymbol{x}}, r\right)=c_{n}^{*}\left(\frac{1}{-\boldsymbol{x}}, r\right)
$$

is Schur-convex on $\mathbb{R}_{-}^{n}$.

If $r$ is an odd integer, then

$$
c_{n}^{*}\left(\frac{1}{\boldsymbol{x}}, r\right)=-c_{n}^{*}\left(\frac{1}{-\boldsymbol{x}}, r\right)
$$

is Schur-concave on $\mathbb{R}_{-}^{n}$.

The proof of Theorem 4 is completed.

By Theorem 4 and majorizing relation (7), it is not difficult to prove the following corollary.

Corollary 1 If $\boldsymbol{x} \in \mathbb{R}_{+}^{n}$ or $r$ is an even integer and $\boldsymbol{x} \in \mathbb{R}_{-}^{n}$, then we have

$$
\prod_{i_{1}+i_{2}+\cdots+i_{n}=r} \sum_{j=1}^{n} i_{j}\left(\frac{1}{x_{j}}\right) \geq\left(\frac{r}{A_{n}(\mathbf{x})}\right)^{\left(\begin{array}{c}
n+r-1 \\
r
\end{array}\right)}
$$

where $A_{n}(\mathbf{x})=\frac{1}{n} \sum_{i=1}^{n} x_{i}$ and $\left(\begin{array}{c}n+r-1 \\ r\end{array}\right)=\frac{(n+r-1) !}{r !((n+r-1)-r) !}$. If $r$ is odd and $\mathbf{x} \in \mathbb{R}_{-}^{n}$, then inequality (10) is reversed. 
Let

$$
c_{n}^{*}\left(\frac{\boldsymbol{x}}{1-\boldsymbol{x}}, r\right)=\prod_{i_{1}+i_{2}+\cdots+i_{n}=r} \sum_{j=1}^{n} i_{j}\left(\frac{x_{j}}{1-x_{j}}\right) .
$$

Theorem 5 The symmetric function $c_{n}^{*}\left(\frac{x}{1-x}, r\right)$ is Schur-convex, Schur-geometrically convex, and Schur-harmonically convex on $\left[\frac{1}{2}, 1\right]^{n}$.

Proof Let $g(x)=\frac{x}{1-x}$. Then $(\ln g(x))^{\prime \prime}=\frac{2 x-1}{x^{2}(1-x)^{2}}$, so $f(x)$ is $\log$-convex on $\left[\frac{1}{2}, 1\right]$; by Theorem $1(\mathrm{a})$, it follows that $c_{n}^{*}\left(\frac{\boldsymbol{x}}{1-\boldsymbol{x}}, r\right)$ is Schur-convex on $\left[\frac{1}{2}, 1\right]^{n}$. Noting that $g(x)$ is increasing on $\left[\frac{1}{2}, 1\right]$, by (a) in Theorem 2 and (a) in Theorem 3, it follows that $c_{n}^{*}\left(\frac{x}{1-x}, r\right)$ is Schurgeometrically convex and Schur-harmonically convex on $\left[\frac{1}{2}, 1\right]^{n}$.

The proof of Theorem 5 is completed.

From the majorizing relation (7), the following majorizing relation is established:

$$
\left(\log G_{n}(\boldsymbol{x}), \log G_{n}(\boldsymbol{x}), \ldots, \log G_{n}(\boldsymbol{x})\right) \prec\left(\log x_{1}, \log x_{2}, \ldots, \log x_{n}\right)
$$

By this majorizing relation and Theorem 5, it is not difficult to prove the following corollary.

Corollary 2 If $\mathbf{x} \in\left[\frac{1}{2}, 1\right]^{n}$, then we have

$$
\prod_{i_{1}+i_{2}+\cdots+i_{n}=r} \sum_{j=1}^{n} i_{j}\left(\frac{x_{j}}{1-x_{j}}\right) \geq\left(\frac{r G_{n}(\mathbf{x})}{1-G_{n}(\mathbf{x})}\right)^{\left(\begin{array}{c}
n+r-1 \\
r
\end{array}\right)},
$$

where $G_{n}(\mathbf{x})=\sqrt[n]{\prod_{i=1}^{n} x_{i}}$.

Let

$$
c_{n}^{*}\left(\frac{1+\boldsymbol{x}}{1-\boldsymbol{x}}, r\right)=\prod_{i_{1}+i_{2}+\cdots+i_{n}=r} \sum_{j=1}^{n} i_{j}\left(\frac{1+x_{j}}{1-x_{j}}\right) .
$$

\section{Theorem 6}

(a) The symmetric function $c_{n}^{*}\left(\frac{1+x}{1-\boldsymbol{x}}, r\right)$ is Schur-convex, Schur-geometrically convex, and Schur-harmonically convex on $(0,1)^{n}$.

(b) If $r$ is an even integer (or odd integer, respectively), then $c_{n}^{*}\left(\frac{1+\boldsymbol{x}}{1-\boldsymbol{x}}, r\right)$ is Schur-convex (or Schur-concave, respectively) on $(1,+\infty)^{n}$.

Proof (a) Let $h(x)=\frac{1+x}{1-x}$. Then $(\ln h(x))^{\prime \prime}=\frac{4 x}{(1+x)^{2}(1-x)^{2}}$, so $f(x)$ is log-convex on $(0,1)$, by Theorem 1(a), it follows that $c_{n}^{*}\left(\frac{1+x}{1-x}, r\right)$ is Schur-convex on $(0,1)^{n}$. Noting that $h(x)$ is increasing on $(0,1)^{n}$, by (a) in Theorem 2 and (a) in Theorem 3, it follows that $c_{n}^{*}\left(\frac{1+x}{1-x}, r\right)$ is Schur-geometrically convex and Schur-harmonically convex on $(0,1)^{n}$.

(b) For $\boldsymbol{x} \in(1,+\infty)$, we consider

$$
c_{n}^{*}\left(\frac{1+\boldsymbol{x}}{\boldsymbol{x}-1}, r\right)=\prod_{i_{1}+i_{2}+\cdots+i_{n}=r} \sum_{j=1}^{n} i_{j}\left(\frac{1+x_{j}}{x_{j}-1}\right) .
$$


Let $h_{1}(x)=\frac{1+x}{x-1}$. Then $\left(\ln h_{1}(x)\right)^{\prime \prime}=\frac{4 x}{(1+x)^{2}(x-1)^{2}}$, so $f(x)$ is log-convex on $(1,+\infty)$, by (a) in Theorem 1, it follows that $c_{n}^{*}\left(\frac{1+\boldsymbol{x}}{\boldsymbol{x}-1}, r\right)$ is Schur-convex on $(1,+\infty)^{n}$.

Noting that

$$
c_{n}^{*}\left(\frac{1+\boldsymbol{x}}{1-\boldsymbol{x}}, r\right)=(-1)^{r} c_{n}^{*}\left(\frac{1+\boldsymbol{x}}{\boldsymbol{x}-1}, r\right),
$$

combining the Schur-convexity of $c_{n}^{*}\left(\frac{1+\boldsymbol{x}}{\boldsymbol{x}-1}, r\right)$, we can get (b) in Theorem 6 .

The proof of Theorem 6 is completed.

Let

$$
c_{n}^{*}\left(\frac{1}{\boldsymbol{x}}-\boldsymbol{x}, r\right)=\prod_{i_{1}+i_{2}+\cdots+i_{n}=r} \sum_{j=1}^{n} i_{j}\left(\frac{1}{x_{j}}-x_{j}\right) .
$$

\section{Theorem 7}

(a) If $r$ is an even integer (or odd integer, respectively), then $c_{n}^{*}\left(\frac{1}{\boldsymbol{x}}-\boldsymbol{x}, r\right)$ is Schur-concave (or Schur-convex, respectively) on $\mathbb{R}_{+}^{n}$.

(b) The symmetric function $c_{n}^{*}\left(\frac{1}{\boldsymbol{x}}-\boldsymbol{x}, r\right)$ is Schur-concave on $\mathbb{R}_{-}^{n}$.

(c) If $r$ is an even integer, then $c_{n}^{*}\left(\frac{1}{\boldsymbol{x}}-\boldsymbol{x}, r\right)$ is Schur-geometrically concave and Schur-harmonically concave on $(-\infty, 1]^{n}$.

Proof First consider

$$
c_{n}^{*}\left(\boldsymbol{x}-\frac{1}{\boldsymbol{x}}, r\right)=\prod_{i_{1}+i_{2}+\cdots+i_{n}=r} \sum_{j=1}^{n} i_{j}\left(x_{j}-\frac{1}{x_{j}}\right) .
$$

(a) Let $p(x)=x-\frac{1}{x}$. Then $p^{\prime \prime}(x)=-\frac{2}{x^{3}}$, so $f(x)$ is concave on $\mathbb{R}_{+}$, by Theorem $1(\mathrm{~b})$, it follows that $c_{n}^{*}\left(\boldsymbol{x}-\frac{1}{\boldsymbol{x}}, r\right)$ is Schur-concave on $\mathbb{R}_{+}^{n}$.

Noting that

$$
c_{n}^{*}\left(\frac{1}{\boldsymbol{x}}-\mathbf{x}, r\right)=(-1)^{n} c_{n}^{*}\left(\boldsymbol{x}-\frac{1}{\boldsymbol{x}}, r\right),
$$

combining the Schur-concavity of $c_{n}^{*}\left(\frac{1}{\boldsymbol{x}}-\boldsymbol{x}, r\right)$, we can get (a) in Theorem 7 .

(b) Noting that

$$
c_{n}^{*}\left(\frac{1}{-\boldsymbol{X}}-(-\mathbf{x}), r\right)=(-1)^{r} c_{n}^{*}\left(\frac{1}{\boldsymbol{x}}-\mathbf{x}, r\right),
$$

combining (a) in Theorem 7 , it is not difficult to verify that (b) in Theorem 7 holds.

(c) It is not difficult to verify that $p(x)=x-\frac{1}{x}$ is nonnegative and decreasing on $(-\infty, 1]$, by Lemma 5 and Lemma 6 , from (a) and (b) in Theorem 7 , it follows that (c) in Theorem 7 holds.

The proof of Theorem 7 is completed.

For $u>1$, let

$$
c_{n}^{*}\left(\frac{u^{\boldsymbol{x}}-1}{\boldsymbol{x}}, r\right)=\prod_{i_{1}+i_{2}+\cdots+i_{n}=r} \sum_{j=1}^{n} i_{j}\left(\frac{u^{x_{j}}-1}{x_{j}}\right) .
$$


Theorem 8 The symmetric function $c_{n}^{*}\left(\frac{u^{\boldsymbol{x}}-1}{\boldsymbol{x}}, r\right)$ is Schur-convex, Schur-geometrically convex, and Schur-harmonically convex on $\mathbb{R}_{+}^{n}$ for $u>1$.

Proof Let $q(t)=\frac{u^{t}-1}{t}$. Then from Lemma 6 and (a) in Theorem 1, it follows that $c_{n}^{*}\left(\frac{u^{\boldsymbol{x}}-1}{\boldsymbol{x}}, r\right)$ is Schur-convex on $\mathbb{R}_{+}^{n}$ for $u>1$.

Since

$$
q^{\prime}(t)=\frac{s(t)}{t^{2}}
$$

where $s(t)=u^{t}(t \log u-1)+1, s^{\prime}(t)=u^{t} \log u \log u^{t}>0$, for $u>1$ and $t>0$, so $s(t) \geq s(0)=0$, and then $q^{\prime}(t) \geq 0$, that is, $q(t)$ is increasing on $\mathbb{R}_{+}^{n}$, by (a) in Theorem 2 and (a) in Theorem 3, it follows that $\left.c_{n}^{*} \frac{u^{\boldsymbol{x}}-1}{\boldsymbol{x}}, r\right)$ is Schur-geometrically convex and Schur-harmonically convex on $\mathbb{R}_{+}^{n}$.

The proof of Theorem 8 is completed.

From the majorizing relation (7), the following majorizing relation is established:

$$
\left(\frac{1}{H_{n}(\boldsymbol{x})}, \frac{1}{H_{n}(\boldsymbol{x})}, \ldots, \frac{1}{H_{n}(\boldsymbol{x})}\right) \prec\left(\frac{1}{x_{1}}, \frac{1}{x_{2}}, \ldots, \frac{1}{x_{n}}\right) .
$$

By this majorizing relation and Theorem 8, it is not difficult to prove the following corollary.

Corollary 3 If $\mathbf{x}=\left(x_{1}, x_{2}, \ldots, x_{n}\right) \in \mathbb{R}_{+}^{n}$ and $u>1$, then

$$
\prod_{i_{1}+i_{2}+\cdots+i_{n}=r} \sum_{j=1}^{n} i_{j}\left(\frac{u^{x_{j}}-1}{x_{j}}\right) \geq\left(\frac{r\left(u^{H_{n}(\mathbf{x})}-1\right)}{H_{n}(\mathbf{x})}\right)^{\left(\begin{array}{c}
n+r-1 \\
r
\end{array}\right)},
$$

where $H_{n}(\mathbf{x})=\frac{n}{\sum_{i=1}^{n} x_{i}^{-1}}$.

Discovering and judging Schur convexity of various symmetric functions is an important subject in the study of the majorization theory. In recent years, many domestic scholars have made a lot of achievements in this field (see [24-30]).

Funding

The paper was supported by the General Project of Science and Technology Plan of Beijing Municipal Education Commission under Grant No. KM202011417012.

Availability of data and materials

Not applicable.

Competing interests

The authors declare that they have no competing interests.

Authors' contributions

The author HS has made the conception of the manuscript and the writing of the first draft, the authors PW and JZ have made the revisions. All authors read and approved the final manuscript.

\section{Publisher's Note}

Springer Nature remains neutral with regard to jurisdictional claims in published maps and institutional affiliations. 


\section{References}

1. Chu, Y.-M., Wang, G.-D., Zhang, X.-H.: The Schur multiplicative and harmonic convexities of the complete symmetric function. Math. Nachr. 284(5-6), 653-663 (2011)

2. Chu, Y.-M., LV, Y.-P.: The Schur harmonic convexity of the Hamy symmetric function and its applications. J. Inequal. Appl. 2009, Article ID 838529 (2009)

3. Xia, W.F., Chu, Y.M.: Schur-convexity for a class of symmetric functions and its applications. J. Inequal. Appl. 2009. Article ID 493759 (2009)

4. Guan, K.-Z.: Schur-convexity of the complete symmetric function. Math. Inequal. Appl. 9(4), 567-576 (2006)

5. Sun, M.B., Chen, N.B., Li, S.H.: Some properties of a class of symmetric functions and its applications. Math. Nachr. (2014). https://doi.org/10.1002/mana.201300073

6. Xia, W.-F., Chu, Y.-M.: Schur convexity and Schur multiplicative convexity for a class of symmetric functions with applications. Ukr. Math. J. 61(10), 1541-1555 (2009)

7. Rovența, I.: Schur convexity of a class of symmetric functions. In: Annals of the University of Craiova. Mathematics and Computer Science Series, vol. 37, pp. 12-18 (2010)

8. Xia, W.-F., Chu, Y.-M.: On Schur convexity of some symmetric functions. J. Inequal. Appl. 2010, Article ID 543250 (2010)

9. Meng, J.-X., Chu, Y.-M., Tang, X.-M.: The Schur-harmonic-convexity of dual form of the Hamy symmetric function. Mat. Vesn. 62(1), 37-46 (2010)

10. Chu, Y.-M., Xia, W.-F., Zhao, T.-H.: Some properties for a class of symmetric functions and applications. J. Math. Inequal. 5(1), 1-11 (2011)

11. Guan, K.-Z., Guan, R.-K.: Some properties of a generalized Hamy symmetric function and its applications. J. Math. Anal. Appl. 376, 494-505 (2011)

12. Qian, W.-M.: Schur convexity for the ratios of the Hamy and generalized Hamy symmetric functions. J. Inequal. Appl. 2011, 131 (2011). https://doi.org/10.1186/1029-242X-2011-131

13. Chu, Y.-M., Xia, W.-F., Zhang, X.-H.: The Schur concavity, Schur multiplicative and harmonic convexities of the second dual form of the Hamy symmetric function with applications. J. Multivar. Anal. 105(1), 412-421 (2012)

14. Rovenţa, I.: A note on Schur-concave functions. J. Inequal. Appl. 2012, 159 (2012). https://doi.org/10.1186/1029-242X-2012-159

15. Xia, W.-F., Zhang, X.-H., Wang, G.-D., Chu, Y.-M.: Some properties for a class of symmetric functions with applications. Indian J. Pure Appl. Math. 43(3), 227-249 (2012)

16. Shi, H.-N., Zhang, J.: Schur-convexity of dual form of some symmetric functions. J. Inequal. Appl. 2013, 295 (2013). https://doi.org/10.1186/1029-242X-2013-295

17. Zhang, K.-S., Shi, H.-N.: Schur convexity of dual form of the complete symmetric function. Math. Inequal. Appl. 16(4), 963-970 (2013)

18. Shi, H.-N., Zhang, J., Ma, Q.-H.: Schur-convexity, Schur-geometric and Schur-harmonic convexity for a composite function of complete symmetric function. SpringerPlus 5, 296 (2016)

19. Zhang, X.-H., Chu, Y.-M.: New Discussion to Analytic Inequalities. Harbin Institute of Technology Press, Harbin (2009) (in Chinese)

20. Shi, H.-N.: Majorization Theory and Analytical Inequalities. Harbin Institute of Technology Press, Harbin (2012) (in Chinese)

21. Shi, H.-N.: Schur-Convex Functions and Inequalities. Harbin Institute of Technology Press, Harbin (2012) (in Chinese)

22. Shi, H.-N., Zhang, J.: Schur-convexity, Schur-geometric and harmonic convexities of dual form of a class symmetric functions. J. Math. Inequal. 8(2), 349-358 (2014)

23. Shi, H.: Schur-Convex Functions and Inequalities: Volume 1: Concepts, Properties, and Applications in Symmetric Function Inequalities. Harbin Institute of Technology Pressd, Harbin (2019)

24. Shi, H.: Schur-Convex Functions and Inequalities: Volume 2: Applications in Inequalities. Harbin Institute of Technology Press, Harbin (2019)

25. Wu, S.-H., Chu, Y.-M.: Schur m-power convexity of generalized geometric Bonferroni mean involving three parameters. J. Inequal. Appl. 2019, Article ID 57 (2019)

26. Xia, W.-F., Chu, Y.-M.: The Schur convexity of Gini mean values in the sense of harmonic mean. Acta Math. Sci. 31B(3), $1103-1112(2011)$

27. Xia, W.-F., Chu, Y.-M., Wang, G.-D.: Necessary and sufficient conditions for the Schur harmonic convexity or concavity of the extended mean values. Rev. Unión Mat. Argent. 52(1), 121-132 (2011)

28. Chu, Y.-M., Wang, G.-D., Zhang, X.-H.: Schur convexity and Hadamard's inequality. Math. Inequal. Appl. 13(4), $725-731$ (2010)

29. Chu, Y.-M., Xia, W.-F., Zhao, T.-H.: Schur convexity for a class of symmetric functions. Sci. China Math. 53(2), 465-474 (2010)

30. Guessab, A., Schmeisser, G.: Sharp integral inequalities of the Hermite-Hadamard type. J. Approx. Theory 115(2) 260-288 (2002)

31. Marshall, A.W., Olkin, I., Arnold, B.C.: Inequalities: Theory of Majorization and Its Application, 2nd edn. Springer, New York (2011)

32. Wang, B.Y.: Foundations of Majorization Inequalities. Beijing Normal University Press, Beijing (1990) (in Chinese)

33. Zhang, X.M.: Geometrically Convex Functions. An'hui University Press, Hefei (2004) (in Chinese)

34. Chu, Y.M., Zhang, X.M., Wang, G.D.: The Schur geometrical convexity of the extended mean values. J. Convex Anal. 15(4), 707-718 (2008)

35. Guan, K.Z.: A class of symmetric functions for multiplicatively convex function. Math. Inequal. Appl. 10(4), 745-753 (2007)

36. Sun, T.-C., LV, Y.-P., Chu, Y.-M.: Schur multiplicative and harmonic convexities of generalized Heronian mean in $n$ variables and their applications. Int. J. Pure Appl. Math. 55(1), 25-33 (2009)

37. Chu, Y.M., Sun, T.C.: The Schur harmonic convexity for a class of symmetric functions. Acta Math. Sci. 30B(5), $1501-1506$ (2010) 